\title{
A Variational Approach to Electrostatics of Polarizable Heterogeneous Substances
}

\author{
Michael Grinfeld ${ }^{1}$ and Pavel Grinfeld ${ }^{2}$ \\ ${ }^{1}$ Aberdeen Proving Ground, US Army Research Laboratory, Aberdeen, MD 21005-5066, USA \\ ${ }^{2}$ Drexel University, Philadelphia, PA 19104, USA \\ Correspondence should be addressed to Michael Grinfeld; michael.greenfield4.civ@mail.mil
}

Received 5 December 2014; Revised 2 April 2015; Accepted 8 April 2015

Academic Editor: Giorgio Kaniadakis

Copyright ( 2015 M. Grinfeld and P. Grinfeld. This is an open access article distributed under the Creative Commons Attribution License, which permits unrestricted use, distribution, and reproduction in any medium, provided the original work is properly cited.

We discuss equilibrium conditions for heterogeneous substances subject to electrostatic or magnetostatic effects. We demonstrate that the force-like aleph tensor $\aleph^{i j}$ and the energy-like beth tensor $\beth^{i j}$ for polarizable deformable substances are divergence-free: $\nabla_{i} \aleph^{i j}=0$ and $\nabla_{i} \beth^{i j}=0$. We introduce two additional tensors: the divergence-free energy-like gimel tensor $\beth^{i j}$ for rigid dielectrics and the general electrostatic gamma tensor $\Gamma^{i j}$ which is not divergence-free. Our approach is based on a logically consistent extension of the Gibbs energy principle that takes into account polarization effects. While the model is mathematically rigorous, we caution against the assumption that it can reliably predict physical phenomena. On the contrary, clear models often lead to conclusions that are at odds with experiment and therefore should be treated as physical paradoxes that deserve the attention of the scientific community.

\section{Introduction}

The goal of this paper is to present a logically consistent extension of the Gibbs variational approach [1] to elastic bodies with interfaces in the presence of electromagnetic effects. Logical consistency and mathematical rigor, in other words, clarity, do not always lead to physical theories that accurately predict experimentally observable phenomena. In fact, Niels Bohr, who stated that clarity is complimentary to truth, may have thought that the clearer the model is, the less likely it is to be a reflection of reality, but, nevertheless, establishing clarity is an essential step along the path towards understanding. This paper pursues clarity and, therefore, poses the acute question of experimental verifiability.

Gibbs suggested building an analysis of equilibrium of heterogeneous substances by analogy with classical statics. He transformed the principle of minimum energy by replacing mechanical energy with internal energy at fixed total entropy. Gibbs' analysis incorporated phase transformations in heterogeneous systems into a general variational framework. Gibbs modeled phase transformations simply as an additional degree of freedom in his variational approach. In the Gibbs analysis, the conditions of phase equilibrium arise as natural boundary conditions (in the sense of variational calculus [2]) corresponding to the additional degree of freedom.

Simplicity was one of Gibbs' primary objectives as he stated it in his own words [3]: "If I have had any success in mathematical physics, it is, I think, because I have been able to dodge mathematical difficulties. Anyone having these desires will make these researches..." Perhaps foreseeing possible misinterpretations of the mathematical implications of his method, Gibbs also wrote [3], "A mathematician may say anything he pleases, but a physicist must be at least partially sane."

Let us now turn to the world of electromagnetism. One of the major achievements of Maxwell's theory [4] was the successful introduction of the stress tensor, originally found in continuum mechanics, to the concept of ether, the agent of electrical and magnetic forces. Historically, Maxwell's theory was not as readily accepted as one might imagine. On the contrary, several leading thinkers, including Helmholtz, rejected his theory either partially or completely. In [5], Poincaré emphasized that certain contradictions are inherent in Maxwell's theory. 
Maxwell himself pointed out a number of difficulties in his theory. Of relevance to this paper is his statement [4]: "I have not been able to make the next step, namely, to account by mechanical considerations for these stresses in dielectrics." Many efforts have since been made to fix this shortcoming. Many of those efforts are variational in nature since one of the most effective ways of coping with mathematical difficulties and logical inconsistencies is to insist on a variational formulation. Among the many textbooks, lectures, and monographs on electromagnetism [6-16], there are many that discuss the variational perspective, and once again it is clear that there is no consensus on the right approach.

One of the pioneers of variational methods in electromagnetism was Gibbs himself. Gibbs studied the problem of equilibrium configuration of charges and discovered that (what researchers now call) the chemical potential of a charged material particle should be supplemented with an additional term $q \varphi$ (attributed by Gibbs to Gabriel Lippmann), where $q$ is the electric charge of the particle and $\varphi$ is the electrostatic potential. This is a very rough sketch of Gibbs' vision. For instance, Gibbs himself has never used the term chemical potential and did not assign the corresponding quantity any profound meaning, which was understood only much later. The variational approach to polarizable substances was most likely pioneered by Korteweg [17] and Helmholtz [18].

Gibbs modeled heterogeneous systems, or what he called heterogeneous substances, as macroscopic domains separated by mathematical surfaces. The difficulty in carrying over Gibbs' ideas to electromagnetism is that the analysis of singular interfaces in electrostatics and magnetostatics is much more challenging than it is in continuum mechanics. Even Lorentz chose to avoid the analysis of heterogeneous systems, stating in the preface to his classical treatise [19] that he does not want to struggle with the boundary terms. Many of the difficulties that were faced (or should have been faced) by Lorentz can be overcome with the help of the calculus of moving surfaces.

In this paper we make a new attempt at extending the Gibbs variational framework to electrostatics. Our approach is very simple and entirely straightforward conceptually. Contrary to many of the prior attempts ([12-14], to name just three), we explicitly exclude the electric field and the electric displacement from the list of independent thermodynamic variables. Instead, we account for polarization (or magnetization) by adding a single term to the "traditional" free energy for a thermoelastic system. The additional term represents the potential energy accumulated in the electrostatic field over the entire space. Different authors choose this term differently: $|\mathbf{E}|^{2}, \mathbf{E} \cdot \mathbf{D}$, and so forth. We choose the integrand in the simplest form $|\mathbf{E}|^{2}$. We build our approach on the exact nonlinear theory of continuum media and rely on Eulerian coordinates as the independent spatial variables.

\section{The Gibbs Thermodynamics in a Nutshell}

According to the modern interpretation of Gibbs, the chemical potential $\mu$ governs the equilibrium between the liquid and the vapor phases with respect to mass exchange between them. Equilibrium heterogeneous systems must satisfy a number of conditions at the phase interface. The first two conditions, those of thermal equilibrium (temperature $T$ is continuous across the interface (and, of course, spatially constant)) and mechanical equilibrium (pressure $p$ is continuous across the interface), are satisfied by all equilibrium twophase systems, whether or not the phases are different states of the same substance subject to a phase transformation. Letting the brackets []$_{-}^{+}$denote the jump discontinuity in the enclosed quantity across the phase interface, we write these conditions as

$$
\begin{aligned}
& {[T]_{-}^{+}=0} \\
& {[p]_{-}^{+}=0 .}
\end{aligned}
$$

Additionally, when the interface is subject to a phase transformation, the chemical potential $\mu$ is continuous across the interface

$$
[\mu]_{-}^{+}=0
$$

This equation is interpreted as equilibrium with respect to mass exchange between the phases. The chemical potential $\mu$ is given by

$$
\mu=\frac{\partial \epsilon(\rho)}{\partial \rho},
$$

where $\rho$ is density and $\epsilon(\rho)$ is the free energy per unit volume.

In many physical systems, equilibrium with respect to mass exchange is attained over much longer time scales than thermal and mechanical equilibria. The dynamics of mass exchange in such systems is often well described by a quasi-static approximation, which assumes that the system maintains thermal and mechanical equilibria throughout evolution; that is, (1) are continuously satisfied, while equilibrium equation (2) is replaced with the following equation for the mass flux $J$ :

$$
J=-K[\mu]_{-}^{+}
$$

where $K>0$ is a kinematic quantity, determined empirically or by some nonthermodynamic theory.

\section{A Variational Approach to Electrostatics of Heterogeneous Systems}

We will now briefly summarize a variational framework for electrostatics of heterogeneous systems which was first described in [20-23]. The presented model, based on the choice of the functional $E$ in (11) and the list of independent variations, is correct only in the mathematical sense; that is, it is logically consistent. Other authors $[10,12,13,24]$ make different choices of energy functionals and sets of independent variations and arrive at different results.

Our description uses the framework of tensor calculus [25]. We refer the space to coordinates $z^{i}$. By convention, we omit the superscript $i$ when the coordinate appears as an argument of a function. We denote the covariant and 


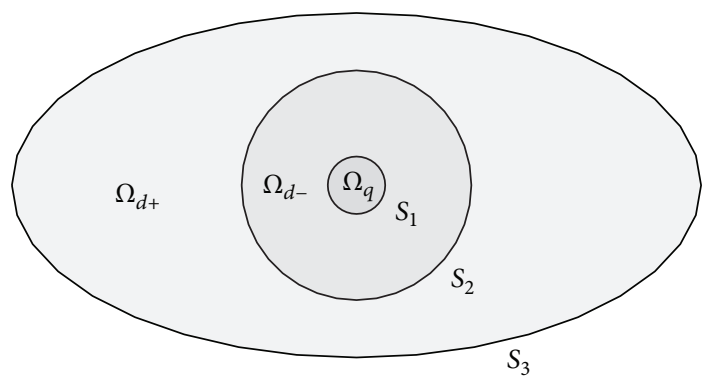

Figure 1: A heterogeneous system with distributed electric charges and dipoles.

contravariant ambient metric tensors by $z_{i j}$ and $z^{i j}$ and the ambient covariant derivative by $\nabla_{i}$.

Figure 1 illustrates the configuration of our system. Suppose that the domain $\Omega_{d}=\Omega_{d+} \cup \Omega_{d-}$ is occupied by solid heterogeneous dielectric media with specific (per unit volume) dipole momentum $P^{i}(z)$. The domain $\Omega_{q}$ is occupied by a stationary electric charge distribution $q(z)$. The two subdomains $\Omega_{d_{+}}$and $\Omega_{d_{-}}$are occupied by two different substances or two different phases of the same substance. They are separated by the interface $S_{2}$.

Suppose that $U^{i}(z)$ is the displacement field of the material particles, $\rho(z)$ is the actual mass density, $\varphi(z)$ is the electrical potential,

$$
E_{i}(z) \equiv-\nabla_{i} \varphi(z)
$$

is the electrical field, and

$$
D_{i}=E_{i}+4 \pi P_{i}
$$

is the electric displacement.

For the sake of simplicity, we assume that the system is kept under fixed absolute temperature $T$ and denote the elastic (internal) energy density $\psi$ of the dielectric substance by

$$
\psi\left(\nabla_{j} U_{i}, P^{k}\right)
$$

Of course, this elastic energy is actually the free energy density of the system.

The equilibrium of the system is governed by Poisson's equation:

$$
\nabla^{i} \nabla_{i} \varphi=4 \pi q
$$

subject to the boundary conditions

$$
\begin{aligned}
{[\varphi]_{-}^{+} } & =0, \\
N^{i}\left[D_{i}\right]_{-}^{+} & =0,
\end{aligned}
$$

across the interfaces ( $N^{i}$ is the unit normal), while at infinity the electrical potential vanishes:

$$
\left.\varphi\right|_{\infty}=0
$$

The total energy $E$ of the system is given by the integral

$$
E=\int\left(\rho \psi+\frac{1}{8 \pi} E_{i} E^{i}\right) d \Omega,
$$

which extends over the entire space.

According to the principle of minimum energy, we associate equilibrium configurations with stationary points of the total energy $E$. In what follows, we use the technique of variation of the energy functionals in the Eulerian description presented in detail in $[21,22,26]$. Suggested procedures for analyzing the equilibrium and stability conditions for twophase heterogeneous systems can be found in [27-30].

We complete the description of the variational principle by presenting the list of quantities treated as the independent variations:

(i) virtual velocity $f^{i}(z)$ of the material particles,

(ii) virtual velocities $C_{2}$ and $C_{3}$ of the interfaces $S_{2}$ and $S_{3}$,

(iii) variation $\delta P^{i}(z)$ of the dipole momentum at the point with coordinates $z^{i}$.

The geometry presented in Figure 1 was analyzed in [21, 28 ] which dealt with nucleation on stationary ions of liquid condensate from the surrounding gaseous phase. When the domain $\Omega_{q}$ is rigid, the virtual velocities of the deformable liquid phase should satisfy the boundary constraint

$$
\left.N_{i} f^{i}\right|_{S_{1}}=0
$$

\section{The Bulk Equilibrium Equations of Deformable Polarizable Substances}

In this section, we summarize the results and refer the reader to the relevant references for the corresponding derivations.

Separating the independent variations in the volume integral of the first energy variation, we arrive at the following equilibrium equations [22, 27]:

$$
\begin{aligned}
-\nabla_{i} \zeta^{i k}+\rho \psi_{P^{i}} \nabla^{k} P^{i} & =0, \\
\rho \psi_{P^{i}} & =E_{i},
\end{aligned}
$$

where $\psi_{P^{i}}=\partial \psi / \partial P^{i}$, the formal stress tensor $\zeta^{m k}$ is defined as

$$
\zeta^{i j} \equiv \rho \frac{\partial \psi}{\partial \nabla_{i} U_{k}} A_{k}^{\cdot j},
$$

and the tensor $A_{\cdot j}^{i \cdot}$ is given by

$$
A_{\cdot j}^{i \cdot} \equiv \delta_{j}^{i}-\nabla_{j} U^{i} .
$$

Combining (13), we arrive at the equilibrium bulk equation

$$
-\nabla_{i} \zeta^{i k}+E_{i} \nabla^{k} P^{i}=0 .
$$

Using the equations of electrostatics, it can be shown that (16) can be rewritten as a statement of vanishing divergence:

$$
\nabla_{i}\left(\zeta^{i j}-z^{i j}\left(\frac{1}{4 \pi} E_{k} D^{k}-\frac{1}{8 \pi} E_{k} E^{k}\right)+\frac{1}{4 \pi} D^{i} E^{j}\right)=0 .
$$


For nonpolarizable substances, the formal stress tensor $\zeta^{i j}$ coincides with the Cauchy stress tensor in the Eulerian description. Relationship (17) generalizes to the celebrated Korteweg-Helmholtz relationship for liquid dielectrics $[6,7$, $10-13,24]$ in the case of nonlinear electroelasticity.

We can rewrite (17) as (see $[22,23,27])$

$$
\nabla_{i} \aleph^{i j}=0
$$

where the aleph tensor $\aleph^{i j}$, given by

$$
\begin{aligned}
\aleph^{i j} \equiv & \rho \frac{\partial \psi}{\partial \nabla_{i} U_{k}} A_{k \cdot}^{\cdot j}+\left(\frac{1}{8 \pi} E_{k} E^{k}-\frac{1}{4 \pi} E_{k} D^{k}\right) z^{i j} \\
& +\frac{1}{4 \pi} D^{i} E^{j}
\end{aligned}
$$

can be thought of as the stress tensor of a polarizable substance. We can rewrite the aleph tensor $\aleph^{i j}$ as

$$
\aleph^{i j} \equiv \zeta^{i j}+\Gamma^{i j},
$$

where the electrostatic gamma tensor $\Gamma^{m k}$ is given by

$$
\Gamma^{i j} \equiv\left(\frac{1}{8 \pi} E_{k} E^{k}-\frac{1}{4 \pi} E_{k} D^{k}\right) z^{i j}+\frac{1}{4 \pi} D^{i} E^{j} .
$$

Equation (17) can be written in another insightful form:

$$
\nabla_{i} \zeta^{i j}=-\nabla_{i} \Gamma^{i j}
$$

In polarizable deformable substances, neither one of the tensors, $\zeta^{i j}$ or $\Gamma^{i j}$, is divergence-free.

The gamma tensor $\Gamma^{i j}$ can be also considered as one of the many possible generalizations of the Maxwell stress tensor $T^{i j}$ :

$$
T^{i j} \equiv-\frac{1}{8 \pi} E_{k} E^{k} z^{i j}+\frac{1}{4 \pi} E^{i} E^{j}
$$

since $\Gamma^{i j}$ coincides with $T^{i j}$ when polarization vanishes. Other possible generalizations of the Maxwell stress tensor,

$$
\begin{aligned}
T_{1}^{i j} & \equiv-\frac{1}{8 \pi} E_{l} D^{l} z^{i j}+\frac{1}{4 \pi} D^{i} E^{j}, \\
T_{2}^{i j} & \equiv-\frac{1}{8 \pi} E_{l} D^{l} z^{i j}+\frac{1}{4 \pi} D^{j} E^{i}, \\
T_{3}^{i j} & \equiv-\frac{1}{8 \pi} E_{l} D^{l} z^{i j}+\frac{1}{8 \pi}\left(D^{i} E^{j}+D^{j} E^{i}\right),
\end{aligned}
$$

are perhaps more aesthetically appealing than the gamma tensor $\Gamma^{i j}$. We believe that the advantage of the gamma tensor over other possible generalizations is its variational origin and its ability to help address the issue of stability based on the calculation of the second energy variation.

One more useful tensor for polarizable materials is the beth tensor $\beth_{\cdot j}^{i}$, or the tensor of electrochemical tensorial potential. It is defined by

$$
\beth_{\cdot j}^{i \cdot} \equiv\left(\rho \psi z^{i k}-\aleph^{i k}+\Gamma^{i k}\right) B_{k j},
$$

where the tensor $B_{k j}$ is the matrix inverse of $A^{k j}$ defined in (15). As we show below, the beth tensor $\beth_{. j}^{i}$ satisfies the condition of zero divergence

$$
\nabla_{i} \beth_{\cdot j}^{i \cdot}=0
$$

similarly to the aleph tensor $\aleph^{i j}$. The beth tensor $\beth_{\cdot j}^{i \cdot}$ can be rewritten as

$$
\beth_{\cdot j}^{i \cdot}=\rho B_{k j} \chi^{i k}
$$

where $\chi^{i k}$ is the Bowen symmetric tensorial chemical potential

$$
\chi^{i j}=\psi z^{i j}-\frac{1}{\rho} \zeta^{i j}=\psi z^{i j}+\frac{1}{\rho}\left(\beth^{i j}-\aleph^{i j}\right) .
$$

The symmetric tensor $\chi^{i j}$ should be distinguished from the typically asymmetric tensorial chemical tensor $\mu^{i j}$ :

$$
\mu^{i j}=z_{\circ}^{k i} z_{l k} \chi^{j l}
$$

where $z_{\circ}^{m i}$ is the contravariant metric tensor of the initial configuration.

\section{Conditions at the Interfaces}

Boundary conditions depend on the various characteristics of the interfaces. Interfaces can differ by their mechanical, or kinematic, properties and whether or not they are subject to phase transformations. We refer to interfaces that satisfy the kinematic constraint

$$
\left[U^{i}\right]_{-}^{+}=0
$$

as coherent interfaces. The following condition for the aleph stress tensor $\mathrm{N}^{i j}$ is satisfied by equilibrium configurations at coherent interfaces:

$$
N_{i}\left[\aleph^{i j}\right]_{-}^{+}=0 .
$$

If, in addition to coherency, the boundary is a phase interface, the condition of phase equilibrium includes the beth tensor $\beth^{i j}$ :

$$
N_{i}\left[\beth^{i j}\right]_{-}^{+}=0
$$

It makes sense, then, to call the beth tensor $\beth^{i j}$ the electrochemical tensorial potential for coherent interfaces in deformable substances because (32) is analogous to the equilibrium condition for the tensorial chemical potential.

\section{Nonfrictional Semicoherent Interfaces}

By definition, nonfrictional semicoherent interfaces are characterized by the possibility of relative slippage. Nonfrictional semicoherent interfaces also may or may not be phase interfaces. Regardless, the following conditions of mechanical equilibrium must hold:

$$
\begin{aligned}
N_{i} \zeta_{ \pm}^{i j} & =-N^{j} p_{ \pm}, \\
N_{i} N_{j}\left[\Gamma^{i j}\right]_{-}^{+} & =[p]_{-}^{+} .
\end{aligned}
$$


At phase nonfrictional incoherent interfaces, an additional mass exchange equilibrium condition must be satisfied:

$$
N_{i} N_{j}\left[\psi z^{i j}+\frac{1}{\rho}\left(\beth^{i j}-\aleph^{i j}\right)\right]_{-}^{+}=0 .
$$

\section{Phase Interfaces in Rigid Dielectrics}

When dealing with rigid solids, all mechanical degrees of freedom disappear and the internal energy depends only on the polarization vector $P^{i}$ (and, unless it is assumed to be constant, temperature $T$ ). At the phase interface, the condition of phase equilibrium reads

$$
N_{i} N_{j}\left[\lambda^{i j}\right]_{-}^{+}=0
$$

where the gimel energy-like tensor $\beth^{i j}$, the electrostatic tensorial chemical potential for rigid dielectrics, is defined by

$$
\beth^{i j} \equiv \epsilon(P) z^{i j}+\Gamma^{i j}
$$

where $\epsilon \equiv \rho \psi$ is the free energy density per unit volume (and we once again suppress the index in $P^{i}$ because it now appears as an argument of a function). We refer to the gimel tensor $\beth^{i j}$ as the electrostatic tensorial chemical potential because it plays the same role as the chemical potential $\mu$ in the classical heterogeneous liquid-vapor system. Contrary to the gamma tensor $\Gamma^{i j}$, the gimel tensor $\beth^{i j}$ is divergence-free:

$$
\nabla_{i} \beth^{i j}=0
$$

One can analyze models in which the polarization vector $P^{i}$ is fixed [20]. Then $\psi_{ \pm}$are spatially constant but may still depend on temperature.

\section{Divergence-Free Tensors in Electrostatics}

We present a proof of the last of the three equations (18), (26), and (37) of vanishing divergence. The remaining two identities can be demonstrated similarly. First, let us rewrite the gimel tensor $\beth^{i j}$ as follows:

$$
\beth^{i j}=\epsilon(P) z^{i j}+\left(\frac{1}{8 \pi} E_{k} E^{k}-\frac{1}{4 \pi} E_{k} D^{k}\right) z^{i j}+\frac{1}{4 \pi} D^{i} E^{j} .
$$

For the first term in (38), we have

$$
\nabla_{i}\left(\epsilon(P) z^{i j}\right)=z^{i j} \frac{\partial \epsilon(P)}{\partial P^{k}} \nabla_{i} P^{k}
$$

Using the thermodynamic identity

$$
\frac{\partial \epsilon(P)}{\partial P^{i}} \equiv E_{i}
$$

we can rewrite (39) as

$$
\nabla_{i}\left(\epsilon(P) z^{i j}\right)=E_{i} \nabla^{j} P^{i} .
$$

For the second term in (38), we have

$$
\begin{aligned}
\nabla_{i} & \left(z^{i j}\left(\frac{1}{8 \pi} E_{k} E^{k}-\frac{1}{4 \pi} E_{k} D^{k}\right)\right) \\
\quad & =-\frac{1}{4 \pi} E_{k} \nabla^{j} E^{k}-P^{k} \nabla^{j} E_{k}-E_{k} \nabla^{j} P^{k},
\end{aligned}
$$

which can be seen from the following chain of identities:

$$
\begin{aligned}
& \text { 2nd term }=\nabla_{i}\left(z^{i j}\left(\frac{1}{8 \pi} E_{k} E^{k}-\frac{1}{4 \pi} E_{k} D^{k}\right)\right) \\
& \begin{aligned}
= & \nabla^{k}\left(\frac{1}{8 \pi} E_{k} E^{k}-\frac{1}{4 \pi} E_{k} D^{k}\right) \\
= & \frac{1}{4 \pi}\left(E_{k} \nabla^{j} E^{k}-\nabla^{j} E_{k} D^{k}-E_{k} \nabla^{j} D^{k}\right) \\
= & \frac{1}{4 \pi}\left(E_{k} \nabla^{j} E^{k}-\nabla^{j} E_{k}\left(E^{k}+4 \pi P^{k}\right)\right. \\
& \left.-E_{k} \nabla^{j}\left(E^{k}+4 \pi P^{k}\right)\right) \\
= & -\frac{1}{4 \pi} E_{k} \nabla^{j} E^{k}-P^{k} \nabla^{j} E_{k}-E_{k} \nabla^{j} P^{k} .
\end{aligned}
\end{aligned}
$$

For the third term in (38), we have

$$
\nabla_{i}\left(\frac{1}{4 \pi} D^{i} E^{j}\right)=\frac{1}{4 \pi} D^{i} \nabla_{i} E^{j}=\frac{1}{4 \pi} E^{i} \nabla_{i} E^{j}+P^{i} \nabla_{i} E^{j} .
$$

Combining (41)-(44), we find

$$
\begin{aligned}
\nabla_{i} \beth^{i j}= & \nabla_{i} \epsilon(P) z^{i j}+z^{i j} \nabla_{i}\left(\frac{1}{8 \pi} E_{l} E^{l}-\frac{1}{4 \pi} E_{l} D^{l}\right) \\
& +\frac{1}{4 \pi} \nabla_{i}\left(D^{i} E^{j}\right) \\
= & E_{i} \nabla^{j} P^{i}-\frac{1}{4 \pi} E_{i} \nabla^{j} E^{i}-P^{i} \nabla^{j} E_{i}-E_{i} \nabla^{j} P^{i} \\
& +\frac{1}{4 \pi} E^{i} \nabla_{i} E^{j}+P^{i} \nabla_{i} E^{j} \\
= & -\frac{1}{4 \pi} E_{i} \nabla^{j} E^{i}-P^{i} \nabla^{j} E_{i}+\frac{1}{4 \pi} E^{i} \nabla_{i} E^{j}+P^{i} \nabla_{i} E^{j} .
\end{aligned}
$$

Finally, using the symmetric property $\nabla_{i} E^{j} \equiv \nabla^{j} E_{i}$, we arrive at identity (37).

\section{Quasi-Static Evolution}

A quasi-static evolution can be postulated by analogy with (4). In the case of nondeformable phases, it reads

$$
J=-K N_{i} N_{j}\left[\beth^{i j}\right]_{-}^{+} .
$$

The same approach can be applied to the case of an isolated domain with fixed total volume yet subject to rearrangement. In this case, the evolution equation should be slightly modified to take into account surface diffusion. Figure 2 illustrates an implementation of this approach in the two-dimensional case. The quasi-static evolution of originally circular domain and fixed polarization vector leads to elongation in the direction of polarization vector $P^{i}$ and, eventually, to a morphological instability. 


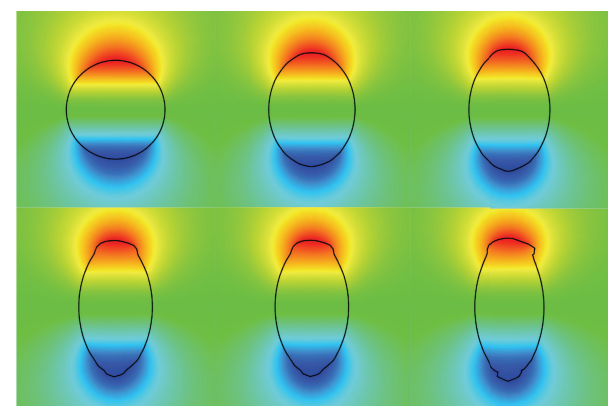

FIGURE 2: Onset of a morphological instability in a quasi-static evolution of a domain filled with dipoles of fixed polarization.

\section{Conclusion}

We discussed a phenomenological variational approach to electrostatics and magnetostatics for heterogeneous systems with phase transformations. Although we focused on electrostatics, almost all of the presented results are also valid for magnetostatics. Our approach is an extension of the Gibbs variational method, as it was interpreted in [26].

The demand of having simultaneously a logically and physically consistent theory remains to be the main driving force of progress in thermodynamics. The suggested approach leads to the mathematically rigorous self-consistent results. Now it has to prove its viability in direct comparison with experiment. That may prove to be difficult, but real progress is only possible when theory and experiment challenge each other.

\section{Appendix}

The summary of notations and variables is as follows (see Abbreviations).

\section{Abbreviations}

$z^{i}$ : $\quad$ Eulerian coordinates in the ambient space

$z_{i j}, z^{i j}$ : Metrics tensors in the reference Eulerian coordinates

$z_{\circ}^{i j}$ : Metrics tensor of the coordinate system, generated by tracking back the coordinate $z^{i}$ from the actual to the initial configuration [26]

$\nabla_{i}: \quad$ The symbols of covariant differentiation (based on the metrics $z_{i j}$ )

$q, P^{i}: \quad$ The electric charge density and polarization (per unit volume)

$\varphi, E^{i}, D^{i}$ : The electrostatic potential, field, and displacement

$\Omega_{q}, \Omega_{d}$ : Spatial domains, occupied by free charges and dipoles

$S_{1}$ : $\quad$ Interface separating the dielectric from the distributed stationary electric charges

$S_{2}$ : $\quad$ Interface separating the different dielectric phases
$S_{3}$ : Interface separating the dielectric phase

from the surrounding vacuum

$U^{i}: \quad$ Displacements of material particles

$A_{\cdot j}^{i \cdot}$ and $B_{\cdot j}^{i \cdot}$ : Mutually inverse geometric tensors defined in (15)

$\rho: \quad$ Mass density

$p, T, \mu: \quad$ Pressure, absolute temperature, and chemical potential of nonpolarizable, one-component liquid phases

$\mu^{i j}, \chi^{i j}$ : Asymmetric and Bowen chemical potentials of nonpolarizable deformable (nonnecessarily liquid) media (for further details, see [26])

$\psi: \quad$ Free energy density per unit mass

$\zeta^{i j}$ : $\quad$ Formal stress tensor defined in (14)

$f^{i}, C_{i}, C_{e}$ : Admissible virtual velocities of the material particles and interfaces

$\aleph^{i j}: \quad$ The aleph tensor, a divergence-free tensor defined in (19); the aleph tensor exhibits some of the properties of the classical Cauchy stress tensor (in Eulerian coordinates) and of the Maxwell stress tensor

$\beth^{i j}$ : $\quad$ The beth tensor, a divergence-free tensor defined in (25); the beth tensor exhibits some of the properties of the scalar chemical potential of nonpolarizable liquid and of the tensorial chemical potentials $\mu^{i j}, \chi^{i k}$ of nonpolarizable solids

$\Gamma^{i j}$ : The gamma tensor defined in (20) for deformable media and in (21) for arbitrary polarizable media

$2^{i j}$ : The gimel tensor which is defined in (36) for rigid dielectrics and plays the same role as the beth tensor $\beth^{i j}$ for deformable dielectrics.

\section{Conflict of Interests}

The authors declare that there is no conflict of interests regarding the publication of this paper.

\section{References}

[1] J. W. Gibbs, "On the equilibrium of heterogeneous substances," Transactions of the Connecticut Academy of Arts and Sciences, vol. 3, pp. 108-248, 1876, vol. 3, pp. 343-524, 1878.

[2] I. M. Gel'fand and S. V. Fomin, Calsulus of Variations, PrenticeHall, Englewood Cliffs, NJ, USA, 1963.

[3] Josiah Willard Gibbs, http://en.wikiquote.org/wiki/Josiah_Willard_Gibbs.

[4] J. C. Maxwell, A Treatise on Electricity and Magnetism, vol. 1-2, Dover Publications, New York, NY, USA, 1954.

[5] H. Poincaré, Lectures on Optics and Electromagnetism. Preface. Collected Papers of Poincaré, vol. 3, Nauka, Moscow, Russia, 1974, (Russian). 
[6] Y. I. Frenkel, Electrodynamics. I. General Theory of Electromagnetism, ONTI, Leningrad, Russia, 1934.

[7] Y. I. Frenkel', Electrodynamics, Volume 2: Macroscopic Electrodynamics of Material Bodies, ONTI, Moscow, Russia, 1935.

[8] I. E. Tamm, Basics of the Theory of Electricity, Nauka, Moscow, Russia, 1989, (Russian).

[9] A. Sommerfeld, Electrodynamics, Academic Press, New York, NY, USA, 1952

[10] J. A. Stratton, Electromagnetic Theory, McGraw-Hill, New York, NY, USA, 1941.

[11] W. K. H. Panofsky and M. Phillips, Classical Electricity and Magnetism, Addison-Wesley, Cambridge, Mass, USA, 1950.

[12] R. A. Toupin, “The elastic dielectric," Indiana University Mathematics Journal, vol. 5, no. 6, pp. 849-915, 1956.

[13] L. D. Landau and E. M. Lifshitz, Electrodynamics of Continuous Media, Pergamon Press, New York, NY, USA, 1963.

[14] I. A. Privorotskiı̆, "Thermodynamic theory of ferromagnetic gomainsins," Soviet Physics Uspekhi, vol. 15, no. 5, pp. 555-574, 1973.

[15] L. I. Sedov and A. G. Tsypkin, Fundamentals of Microscopic Theories of Gravitation and Electromagnetism, Nauka, Moscow, Russia, 1989.

[16] R. E. Rosensweig, Ferrohydrodynamics, Dover, New York, NY, USA, 1985.

[17] D. J. Korteweg, "Über die veränderung der form und des volumens dielectrischer Körperunter Einwirkung elektrischer Kräfte," Annalen der Physik und Chemie, vol. 245, no. 1, pp. 4861,1880 .

[18] H. Helmholtz, "Über die auf das Innere magnetisch oder dielectrisch polarisirter Körper wirkenden Kräfte," Annalen der Physik, vol. 249, no. 7, pp. 385-406, 1881.

[19] H. A. Lorentz, The Theory of Electrons: and Its Applications to the Phenomena of Light and Radiant Heat, Dover, New York, NY, USA, 2011.

[20] M. A. Grinfeld, "Morphology, stability, and evolution of dipole aggregates," Proceedings of the Estonian Academy of Sciences, Engineering, vol. 5, no. 2, pp. 131-141, 1999.

[21] P. Grinfeld, "Morphological instability of liquid metallic nuclei condensing on charged inhomogeneities," Physical Review Letters, vol. 87, no. 9, Article ID 095701, 4 pages, 2001.

[22] M. Grinfeld and P. Grinfeld, "Towards thermodynamics of elastic electric conductors," Philosophical Magazine A, vol. 81, no. 5, pp. 1341-1354, 2001.

[23] M. A. Grinfeld and P. M. Grinfeld, "The exact conditions of thermodynamic phase equilibrium in heterogeneous elastic systems with dipolar interaction," in Nonlinear Mechanics, L. M. Zubov, Ed., pp. 47-51, Rostov University, 2001.

[24] M. Abraham and R. Becker, The Classical Theory of Electricity and Magnetism, Blackie \& Son, 1932.

[25] P. Grinfeld, Introduction to Tensor Analysis and the Calculus of Moving Surfaces, Springer, New York, NY, USA, 2014.

[26] M. Grinfeld, Thermodynamic Methods in the Theory of Heterogeneous Systems, Interaction of Mechanics and Mathematics Series, Longman Scientific \& Technical, Harlow, UK, 1991.

[27] P. Grinfeld and M. Grinfeld, "Thermodynamic aspects of equilibrium shape and growth of crystalline films with electromechanical interaction," Ferroelectrics, vol. 342, no. 1, pp. 89$100,2006$.

[28] P. Grinfeld, "Morphological instability of the dielectric thomson nuclei," Physical Review B, vol. 81, no. 18, Article ID 184110, 2010.
[29] P. Grinfeld, "Clausius-Clapeyron relations for an evaporating solid conductor," Zeitschrift für Angewandte Mathematik und Mechanik, vol. 90, no. 7-8, pp. 633-640, 2010.

[30] P. Grinfeld, "A proposed experiment for the verification of Thomson's nucleation theory," Ferroelectrics, vol. 413, no. 1, pp. 65-72, 2011. 


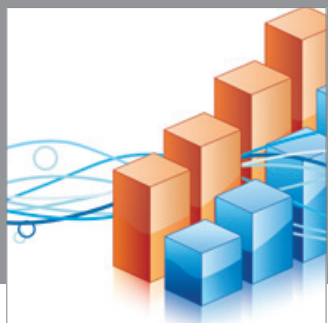

Advances in

Operations Research

mansans

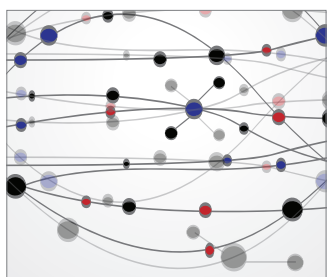

The Scientific World Journal
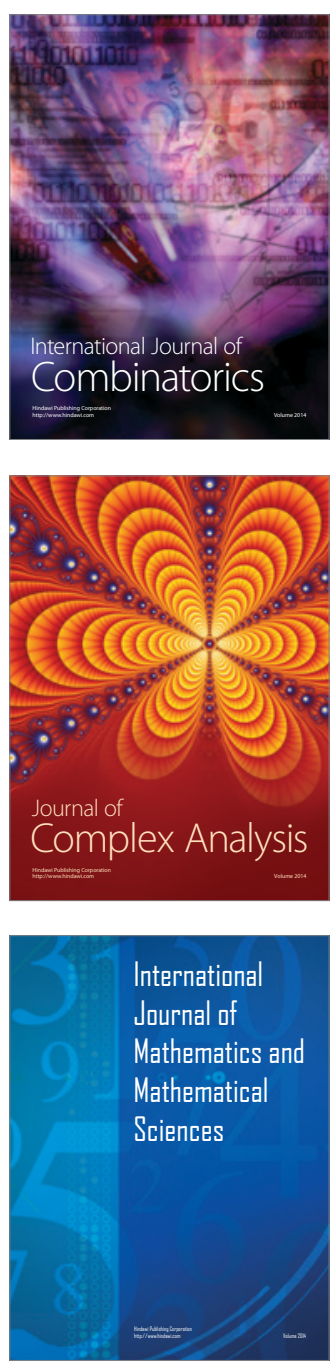
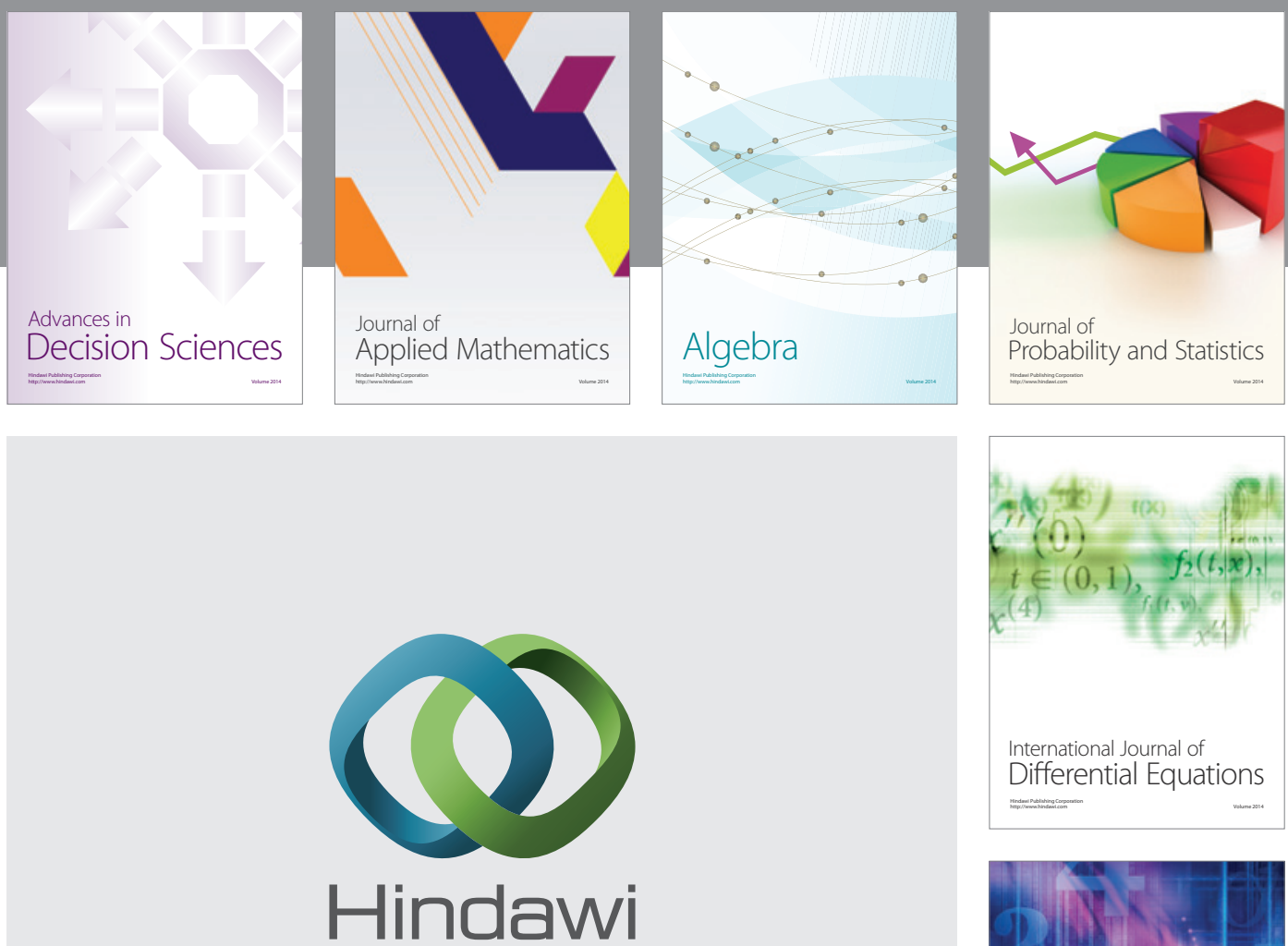

Submit your manuscripts at http://www.hindawi.com
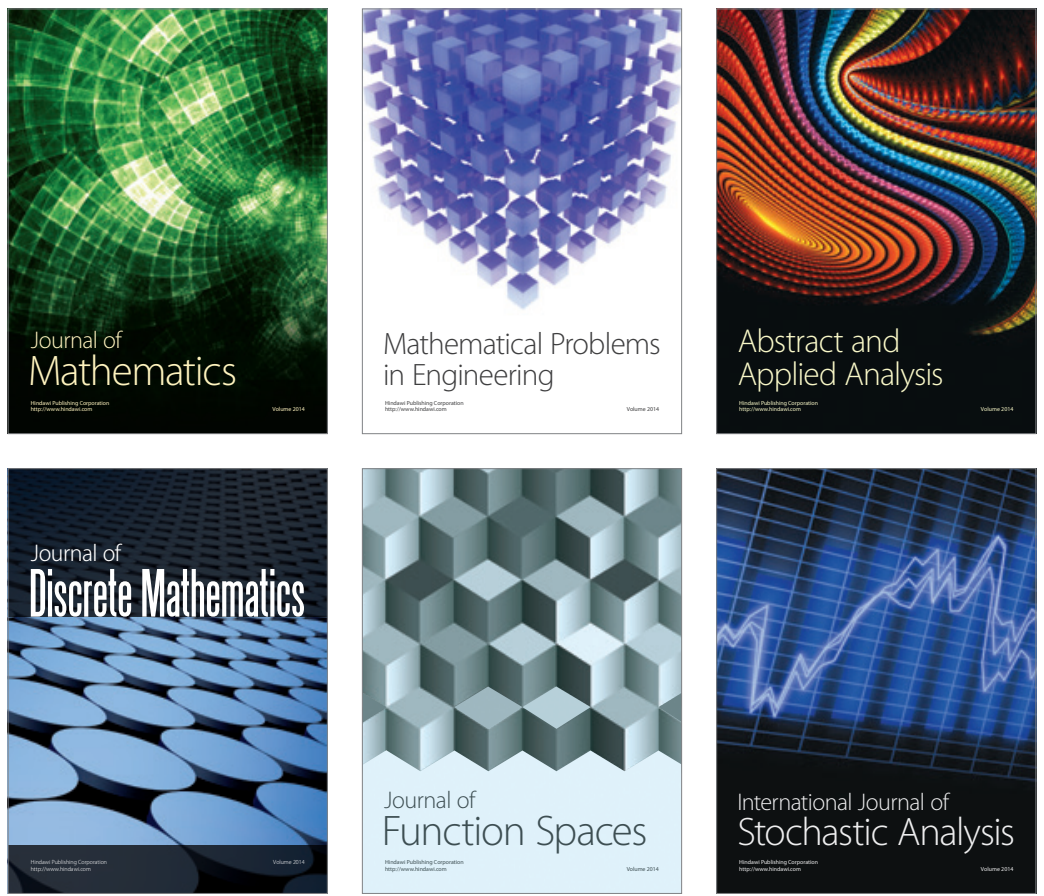

Journal of

Function Spaces

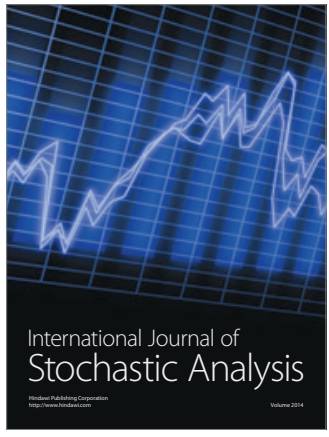

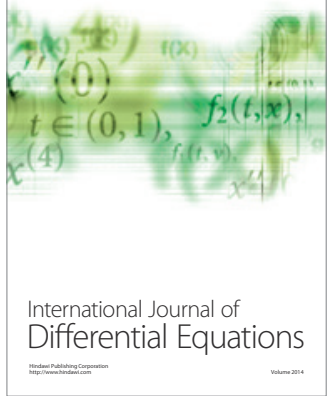
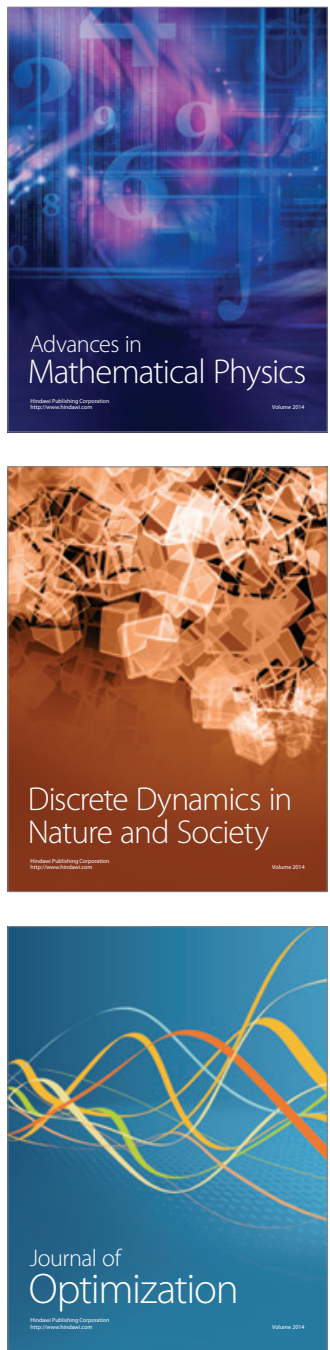